\title{
Non-rectifiable limit sets of dimension one
}

Christopher J. Bishop

\begin{abstract}
We construct quasiconformal deformations of convergence type Fuchsian groups such that the resulting limit set is a Jordan curve of Hausdorff dimension 1, but having tangents almost nowhere. It is known that no divergence type group has such a deformation. The main tools in this construction are (1) a characterization of tangent points in terms of Peter Jones' $\beta$ 's, (2) a result of Stephen Semmes that gives a Carleson type condition on a Beltrami coefficient which implies rectifiability and (3) a construction of quasiconformal deformations of a surface which shrink a given geodesic and whose dilatations satisfy an exponential decay estimate away from the geodesic.
\end{abstract}

\section{Introduction}

In [6] Bowen proved that any deformation of a cocompact Fuchsian group gives a quasi-Fuchsian Kleinian group whose limit set is either a circle or has Hausdorff dimension $>1$. This was extended to all divergence type groups by the author in [3] and was shown to be false for all convergence groups (of the first kind) by Astala and Zinsmeister in [1]. They showed that all such groups have a deformation such that the limit set is a non-circular rectifiable curve.

Zinsmeister asked if Bowen's property could fail in a different way, namely, are there quasi-Fuchsian groups whose limit sets are not locally rectifiable, but still have dimension 1? We will show there are many such groups.

2000 Mathematics Subject Classification: Primary: 30F60.

Keywords: Hausdorff dimension, quasi-Fuchsian groups, quasiconformal deformation, critical exponent, convex core. 
Theorem 1.1. If $R=\mathbb{D} / G$ is a Riemann surface with Green's function (i.e., $G$ is convergence type) and with bounded injectivity radius, then there is a quasiconformal deformation of $G$ whose limit set has Hausdorff dimension 1 and has tangents on a set of at most 1-dimensional measure zero (and hence it contains no rectifiable subarcs). In fact, given any function $\varphi(t)=o(t)$, we can choose such a deformation $f_{\mu}$ so that $\Lambda=f_{\mu}(\mathbb{T})$ has zero $\varphi$-Hausdorff measure.

Note that if $R$ has bounded injectivity radius then $G$ is of the first kind, i.e., the limit set is the whole circle. A Fuchsian group $G$ is called convergence type if $\sum_{g \in G}(1-|g(0)|)<\infty$, and otherwise it is called divergence type. It is well known that $G$ is convergence type iff $R=\mathbb{D} / G$ has a Green's function. A conformal mapping $f: \mathbb{D} \rightarrow \Omega$ is called a deformation of the Fuchsian group $G$ if for every $g \in G, f \circ g \circ f^{-1}$ is Möbius transformation restricted to $\Omega$. It is called a quasiconformal deformation if $f$ has a quasiconformal extension to the whole plane. The corresponding Beltrami coefficient is $\mu=f_{\bar{z}} / f_{z}$ and has $L^{\infty}$ norm $k=\|\mu\|_{\infty}<1$ if $f$ is quasiconformal. The number $K=(k+1) /(k-1)$ is called the quasiconformal constant of $f$. If $\mu$ is a $G$ invariant coefficient with $\|\mu\|_{\infty}<1$ on $\mathbb{D}^{*}=\{|z|>1\} \cup\{\infty\}$, i.e., satisfies

$$
\mu(g(z))=\mu(z) g^{\prime}(z) /\left|g^{\prime}(z)\right|
$$

for all $g \in G$, then there is a corresponding quasiconformal deformation which we will denote $f_{\mu}$ (unique up to Möbius transformations). Note that for such a $\mu,|\mu|$ is well defined on the quotient surface. The injectivity radius of $G$ at $z \in \mathbb{D}$ is $\operatorname{inj}(z)=\frac{1}{2} \rho\left(z, G^{*}(z)\right)$, where $G^{*}(z)$ denotes all the images of $z$ under non-identity elements of $G$.

If $\Omega$ is simply connected, a point $x \in \partial \Omega$ is called an inner tangent point if for every $\theta<\pi, x$ is the vertex of a cone in $\Omega$ of angle $\theta$ and this is not true for any $\theta>\pi$. If $\partial \Omega=\Gamma$ is a Jordan curve then we say $x$ is a tangent point iff it is an inner tangent for both sides of $\Gamma$. If $\Gamma$ is a quasicircle then $x$ being an inner tangent (for either side) is equivalent, up to sets of zero linear measure, to $x$ being a tangent point (e.g., [12]).

The support of the Beltrami coefficient $\mu$ in Theorem 1.1 cannot be compact modulo $G$. If it were, then results of [4] imply that $\Lambda=f_{\mu}(\mathbb{T})$ has tangents on a set of positive linear measure (indeed, on full harmonic measure).

A generalized $Y$-piece in $R$ is a region bounded by three closed geodesics (or punctures) which is homeomorphic to a 2-sphere minus three disks (or points). An $X$-piece in $R$ is the union of two (not necessarily distinct) $Y$-pieces which share a common boundary geodesic $\gamma$, which is called the central curve of $X$. The following is proven in [2]. 
Lemma 1.2. Given an L-bounded $X$-piece $X_{1}$, its central curve $\gamma$ and any $0<\epsilon<1 / 2$, there is an $X$-piece $X_{2}$ and a quasiconformal map $f: X_{1} \rightarrow X_{2}$ so that $f(\gamma)$ is the central geodesic for $X_{2}$ and has length $1-\epsilon$ times that of $\gamma$. Moreover, $f$ is an isometry on the boundary of $X_{1}$ and the corresponding Beltrami coefficient $\mu_{\gamma, \epsilon}$ satisfies $\left\|\mu_{\gamma, \epsilon}\right\|_{\infty}<C \epsilon$ and

$$
\left|\mu_{\gamma, \epsilon}(z)\right| \leq C \epsilon \exp (-2 \operatorname{dist}(z, \gamma)) .
$$

Such $\mu_{\gamma, \epsilon}$ 's will be the building blocks of the desired Beltrami coefficient $\mu$. The proof of this estimate is only difficult in the case when $\gamma$ is extremely short, in which case the $X$-piece has large diameter. Moreover, $\mu_{\gamma, \epsilon}$ is supported on the union of the thin part associated to $\gamma$ (if any) and the two central pieces of the $Y$-pieces making up $X$ (see Section 6 of [2]). This union will be called the core of the $X$-piece.

Suppose $R$ is a Riemann surface and $R=\mathbb{D} / G$, where $G$ is a Fuchsian group. We assume that the injectivity radius of $R$ is bounded above by some $M<\infty$. The goal in this paper is to construct a $G$-invariant Beltrami coefficient $\mu$ on $\mathbb{D}^{*}$, so that $f_{\mu}(\mathbb{T})$ has Hausdorff dimension 1 , but has tangents almost nowhere (with respect to linear measure). If we also assumed that there was a positive lower bound for the injectivity radius, then the argument would be easier in the sense that Lemma 1.2 would be much easier. Certain other cases (e.g., some infinite covers of a compact surface) are even simpler and could be used if one only wanted a single example as in Theorem 1.1.

The first step is to show that a $X$-piece can be found near any point we want. For example, we will show

Lemma 1.3. Suppose $R$ is a Riemann surface such that the injectivity radius is bounded above by $M<\infty$ at every point. Then there is a $L=L(M)<\infty$ so that for every point $z \in R$ there is a $Y$-piece $Y \subset R$ such that each boundary arc of $Y$ has length $\leq L$ and either $z \in Y$ or the distance from $z$ to the thick part of $Y$ is $\leq L$.

Using this, we can make collections of pairwise disjoint $X$-pieces which have large harmonic measure with respect to any given point of $R$. More precisely, given a point $z_{n} \in R$ and compact set $K_{n}$, we will show that we can find a disjoint collection of $X$-pieces $\left\{X_{n, j}\right\}$ with central curves $\left\{\gamma_{n, j}\right\}$, which all lie outside $K_{n}$ and which have large harmonic measure with respect to $z_{n}$, i.e., there is a $\delta>0$ (depending only on $M$, the injectivity upper bound) so that

$$
u_{n}\left(z_{n}\right) \equiv \omega\left(z_{n}, \cup_{j} \gamma_{n, j}, R \backslash \cup_{j} \gamma_{n, j}\right) \geq \delta
$$


Given such a collection, we set $\epsilon_{n, k}=\omega\left(z_{n}, \gamma_{n, k}, R \backslash \cup_{j} \gamma_{n, j}\right)$ and let $\mu_{n, j}$ be the Beltrami coefficient supported on $X_{j}$ which shrinks the length of $\gamma_{n, j}$ by a factor of $1-\sqrt{\epsilon \epsilon_{n, j}}$. We then define $\mu_{n}=\sum_{j} \mu_{n, j}$ and $\mu=\sum_{n} \mu_{n}$.

This definition may look a bit mysterious, but it is quite easy to motivate. In order to show that $f_{\mu}(\mathbb{T})$ has dimension one, we are going to choose each $\mu_{n}$ so that $f_{\mu_{n}}(\mathbb{T})$ is rectifiable. To prove that $f_{\mu_{n}}(\mathbb{T})$ is rectifiable, we will use a theorem of Stephen Semmes [13] that this happens if

$$
\left|\mu_{n}(z)\right|^{2} \frac{d x d y}{1-|z|^{2}}
$$

is a Carleson measure of small norm. One can show this is a Carleson measure if

$$
\sup _{w \in \mathbb{D}} \int_{\mathbb{D}}\left|\mu_{n}(z)\right|^{2} \tilde{g}(z, w) \frac{d x d y}{\left(1-|z|^{2}\right)^{2}}<\infty
$$

where $\tilde{g}(z, w)=\log \left|\frac{1-\bar{w} z}{w-z}\right|$ is the Green's function for the disk. Since $\left|\mu_{n}\right|$ is $G$-invariant, this integral is the same as

$$
\sup _{w \in R} \int_{R}\left|\mu_{n}(z)\right|^{2} g(z, w) d A(z),
$$

where $g$ is the Green's function for $R$ and $d A$ denotes hyperbolic area on $R$. However, this integral (as a function of $w$ ) gives a superharmonic function on $R$ whose Laplacian is $-\left|\mu_{n}\right|^{2}$. Thus to make it bounded we want $\left|\mu_{n}\right|^{2}$ to look like the Laplacian of a bounded subharmonic function on $R$. This is exactly what our definition of $\mu_{n}$ above does (it is an approximation of $-\Delta u_{n}$, where $u_{n}$ is the harmonic measure of $\left.R \backslash \cup_{j} \gamma_{n, j}\right)$.

Taking $\left\{z_{n}\right\}$ to be a dense sequence in $R$ and $K_{n}$ to be a closed $r_{n^{-}}$ neighborhood of $\cup_{k \leq n} \cup_{j} X_{k, j}$ we inductively define a sequence of Beltrami coefficients $\left\{\mu_{n}\right\}$ whose supports are far apart and leave every compact set. Using the estimates for the building block $\mu_{n, j}$ 's and the definition of $\epsilon_{n, j}$, we can show that

$$
\sup _{w} \int_{R} \mu_{n}^{2}(z) g(z, w) d A(z)<C \epsilon
$$

and hence that

$$
\frac{\left|\mu_{n}(z)\right|^{2} d x d y}{1-|z|^{2}},
$$

is a Carleson measure on the unit disk of norm at most $C \epsilon$. Thus by Semmes' theorem, for any fixed $n, f_{\mu_{n}}(\mathbb{T})$ is rectifiable. Combined with the fact that the supports of $\left\{\mu_{n}\right\}$ can be chosen as far apart as we wish, we can then deduce that $f_{\mu}(\mathbb{T})$ has dimension 1 . 
On the other hand, we will show that shrinking a closed geodesic $\gamma$ by a factor of $1-\epsilon$ implies that the corresponding limit set must "wiggle" by a factor of at least $C \epsilon$ when viewed from a point on the geodesic. We will make this idea precise in terms of Peter Jones' $\beta$ 's which measure the deviation of a set from straight line. Jones defines

$$
\beta_{\Gamma}(x, t)=\inf _{L} \frac{1}{t} \sup _{z \in \Gamma \cap B(x, t)} \operatorname{dist}(z, L),
$$

where $L$ is the set of all lines hitting $x$. It follows from results in [5] that if $\Gamma$ is a quasicircle, then up to sets of zero 1-dimensional measure, $x$ is a tangent point of $\Gamma$ iff

$$
\int_{0}^{\infty} \beta_{\Gamma}(x, t)^{2} \frac{d t}{t}<\infty
$$

For our purposes, it is more convenient to introduce a Möbius invariant version of the $\beta$ 's, namely,

$$
\eta_{\Gamma}(z)=\min \left(1, \inf _{\tau} \sup _{x, y \in \Gamma} \log \frac{|\tau(x)|}{|\tau(y)|}\right)
$$

where the infimum is over all Möbius transformations $\tau$ such that $\tau(z)=0$. In other words, $\eta$ measures the smallest $r$ such that $\Gamma$ can be mapped in $\{1 \leq|w| \leq r\}$ with $z$ going to the origin. It is easy to bound $\eta$ in terms of a sum of $\beta$ 's over different scales and to give an almost everywhere criteria for tangent points in terms of $\eta$.

The advantage of $\eta$ is that it is clearly invariant under Möbius transformations, so if $\Gamma$ is the limit set of a Kleinian group, then $\eta(z)$ is a well defined function on the quotient Riemann surface. Thus one can think of $\eta$ as a geometric version of the Schwarzian derivative. We shall prove that if $\Gamma$ is the limit set of a quasi-Fuchsian group corresponding to a Riemann surface $R_{1}$ on one side and its quasiconformal image $R_{2}$ on the other and if $\gamma_{1}$ is a closed geodesic in $R_{1}$ whose image is closed geodesic $\gamma_{2}$ in $R_{2}$ of length $(1+\epsilon) \ell\left(\gamma_{1}\right)$, then $\eta \geq C \epsilon$ at all points in a unit neighborhood of $\gamma_{1}$. In other words, changing the length of a closed geodesic by a definite amount causes a "wiggle" in the limit set of comparable size.

The calculation that shows $\mu_{n}$ corresponds to a Carleson measure of norm at most $C \epsilon$ also shows that its Carleson norm is comparable to $\epsilon$. Thus $\mu$ is a sum of measures whose Carleson norms are uniformly bounded away from zero. Moreover, the closed geodesics $\left\{\gamma_{n, j}\right\}$ chosen to have large harmonic measure from $z_{n}$ and $\left\{z_{n}\right\}$ was chosen to be dense in $R$. Using these facts, 
we will show that if $\Gamma=f_{\mu}(\mathbb{T})$, then

$$
\int_{0}^{1} \eta_{\Gamma}\left(f_{\mu}\left(r e^{i \theta}\right)\right)^{2} \frac{d r}{1-r}=\infty
$$

for almost every $\theta$. This and the comparison between and $\eta$ and $\beta$ allows us to deduce that the integral in (1.2) diverges almost everywhere and hence that almost no point of $\Gamma=f_{\mu}(\mathbb{T})$ is a tangent point.

Next we describe the structure of the rest of the paper. It splits roughly into three pieces: Sections 3-5 which show there are $X$-pieces near any point and collections of $X$-pieces with large harmonic measure; Sections 6-9 which prove that $f_{\mu}(\mathbb{T})$ has tangents almost nowhere; and Sections 10-12 which prove that $f_{\mu}(\mathbb{T})$ has dimension 1 . More precisely,

Section 2 We give a few estimates for Green's function on $R$.

Section 3 We prove that if $R$ has injectivity radius bounded above by $M$, then every point $z \in R$ is either in a $Y$-piece with all boundary lengths $\leq L(M)$ or is a bounded hyperbolic distance from the thick part of such a $Y$-piece.

Section 4 Given a point $z \in R$ and a compact $K \subset R$ we construct a collection of disjoint $X$-pieces which all lie outside $K$, but which have large harmonic measure with respect to $z$.

Section 5 We use the deformations of $X$-pieces described earlier to define the desired dilatation $\mu=\sum \mu_{n}$.

Section 6 We prove a lemma about Stolz integrals of Carleson measures.

Section 7 We recall Peter Jones' $\beta$ 's and introduce a Möbius invariant version $\eta$. The divergence of a certain integral involving $\eta$ implies a.e. that a point is not a tangent point.

Section 8 We show that a deformation which shortens a simple closed geodesic by a factor of $\epsilon$ forces $\eta(z)>C \epsilon$ at points near the geodesic.

Section 9 We show that $f_{\mu}(\mathbb{T})$ has tangents almost nowhere.

Section 10 We subdivide the disk into disjoint chord-arc domains, each of which intersects the support of at most one of the $\mu_{n}$ 's.

Section 11 We recall a theorem of Steven Semmes about quasiconformal mappings of chord-arc domains and prove that our $\mu$ corresponds to a Carleson measure with respect to the stopping time domains of the previous section.

Section 12 We prove that $f_{\mu}(\mathbb{T})$ has dimension 1 . 
I thank Michel Zinsmeister for his comments and questions on [3] which led me to consider the questions dealt with here. I also thank the two referees whose careful reading and numerous suggestions were greatly appreciated.

If $A, B$ are quantities that depend on some parameter we write $A \lesssim B$ if the ratio $B / A$ is bounded uniformly independent of the parameter. We will also sometimes write this as $A=O(B)$. Similarly for $\gtrsim$. We write $A \simeq B$ if both $A \lesssim B$ and $A \gtrsim B$ hold and say $A$ and $B$ are comparable.

\section{Estimates for Green's function}

In this section we record some simple estimates related involving the Green's function for $R=\mathbb{D} / G$ and Beltrami coefficients satisfying the exponential decay estimate (1.1). Most of what we need follows from two easy facts. First, Harnack's inequality, which says that if $u$ is a positive harmonic function on the unit disk then

$$
u(x) \leq C e^{\rho(x, y)} u(y) .
$$

Second, the observation that if $x \in R$ lifts to the origin in the disk, then

$$
\alpha(x) \equiv \operatorname{area}(\{z \in R: \rho(x, z) \leq 1\}) \simeq \min (1, \operatorname{inj}(x)) \simeq \frac{1}{N},
$$

where $N$ is the number of $G$-images of 0 in the hyperbolic ball $B_{\rho}(0,1)$. The estimate can be proven by first noting that there is nothing to do (except take a large enough constant) if the injectivity radius at $x$ is bounded away from zero, $\operatorname{say} \operatorname{inj}(x) \geq \epsilon_{M}>0$, where $\epsilon_{M}$ denotes the Margulis constant. On the other hand if $\operatorname{inj}(x)<\epsilon_{M}$, then $x$ is in a thin part of $R$ and the only $G$-images of 0 near 0 correspond to powers of the associated parabolic or hyperbolic element $g \in G$, and the desired estimate follows easily.

Lemma 2.1. Suppose $g$ is the Green's function for a hyperbolic Riemann surface. Then there is $C_{1}$, such that for any $x, w \in R$,

$$
\frac{1}{C_{1}} \leq \frac{\int_{B(x, 1)} g(z, w) d A(z)}{\alpha(x) \min _{z \in B(x, 1)} g(z, w)} \leq C_{1} .
$$

Proof. This is simply Harnack's inequality if $\rho(x, w) \geq 2$, so we may assume $\rho(x, w)<2$. To prove $(2.1)$, lift $g$ to the disk so that the point $x$ lifts to the origin. Then

$$
g(z, x)=\sum_{n} \tilde{g}\left(z, w_{n}\right)
$$


where $\left\{w_{n}\right\}$ is an enumeration of the orbit of zero and

$$
\tilde{g}(z, w)=\log \left|\frac{1-\bar{w} z}{z-w}\right|
$$

is the Green's function for the disk. Split the sum into two terms, $g_{1}+g_{2}$, corresponding to the $w_{n}$ 's which are either more than or less than hyperbolic distance 2 from the origin. The sum $g_{1}$ over the "far" points is harmonic on a unit hyperbolic ball around the origin and so (2.1) holds by Harnack's inequality. The number $N$ of orbits inside the ball of radius 2 is bounded by a constant divided by the injectivity radius at $x$ and it is easy to see that

$$
\int_{B(0,1)} g_{1} d A \simeq N, \quad \min _{B(0,1)} g_{1} \simeq N
$$

which proves the desired inequalities since $\alpha(x)$ is the area in $R$ of $B(x, 1)$ $\subset R$.

Lemma 2.2. Suppose $g$ is the Green's function for a hyperbolic Riemann surface. Then there is $C_{2}$, such that for any $x, y, w \in R$, such that $1 \leq$ $\rho(w, x) \leq 4,1 \leq \rho(w, y) \leq 4$,

$$
\frac{1}{C_{2}} \leq \frac{g(x, w)}{g(y, w)} \leq C_{2}
$$

Proof. The proof is almost the same as the proof of (2.1). Lift $g$ to the disk so the pole lifts to the origin and divide the sum according to whether $w_{n}$ is further than distance 5 from the origin or closer than distance 5 . For the first sum the result holds by Harnack's inequality. For the second sum it holds because the sum is comparable to $N$ (the number of terms in the sum) at both $x$ and $y$.

Lemma 2.3. Suppose $g$ is the Green's function for a hyperbolic Riemann surface. Then there is $C_{3}$, such that for any $x, y, w \in R$,

$$
\min _{z \in B(x, 1)} g(z, w) \leq C_{3} e^{\rho(x, y)} \min _{z \in B(y, 1)} g(z, w) .
$$

Proof. There are a few cases, depending on the location of the pole $w$ with respect to $x$ and $y$. If $\rho(y, w) \geq \rho(y, x)+1$, then the desired inequality follows directly from Harnack's inequality since $g$ is positive and harmonic on $\{z: \rho(y, z)<\rho(y, w)\}$.

If $\rho(w, y) \leq 1$ then choose a point $z_{1}$ on the geodesic from $y$ to $x$ which is distance 2 from $y$. Then by Harnack's inequality and (2.2),

$$
\min _{z \in B(1, x)} g(z, w) \leq C g(x, w) \leq C e^{\rho\left(x, z_{1}\right)} g\left(z_{1}, w\right) \leq C e^{\rho(x, y)} \min _{z \in B(y, 1)} g(z, w) .
$$


Similarly if $w$ is close to $x$. Finally, if $w$ is more than distance 1 from both $x$ and $y$, we can choose two points $z_{1}, z_{2}$ on the geodesic from $y$ to $x$ such that

$$
\begin{aligned}
\rho\left(z_{1}, z_{2}\right) \leq 2 & \\
\rho\left(z_{1}, w\right) & \geq 1, \\
\rho\left(y, z_{1}\right) & \leq \rho\left(z_{2}, w\right) \geq 1, \\
& \quad \rho\left(x, z_{2}\right) \leq \rho(x, w)-1,
\end{aligned}
$$

This is an easy exercise with the triangle inequality. Just as above we deduce that

$$
\begin{gathered}
g(y, w) \geq C e^{-\rho\left(y, z_{1}\right)} g\left(z_{1}, w\right), \\
g(x, w) \leq C e^{\rho\left(x, z_{2}\right)} g\left(z_{2}, w\right) .
\end{gathered}
$$

Thus it suffices to show the values at $z_{1}$ and $z_{2}$ are comparable, but this follows from (2.2) and Harnack.

Lemma 2.4. Suppose $Y \subset R$ is a L-bounded $Y$-piece and $g$ is the Green's function for $R$. Suppose $\mu$ is bounded function which satisfies the estimate (1.1) with respect to a boundary geodesic $\gamma \subset \partial Y$. Then for any $w \in R$

$$
\int_{Y}|\mu(z)|^{2} g(z, w) d A(z) \leq C \epsilon^{2} \int_{\gamma} g(z, w) d \sigma(z),
$$

where $d \sigma$ denotes hyperbolic arclength measure.

Proof. As functions of $w$, the integrals are both harmonic off $Y$ and tend to zero at infinity. Thus we need only consider $w \in Y$ by the maximum principle. By Lemma 2.1,

$$
\int_{B(x, 1)} g(z, w) d A(z) \simeq \operatorname{inj}(x) \min _{B(x, 1)} g(z, w) .
$$

By Lemma 2.3

$$
\min _{B(x, 1)} g(z, w) \leq C e^{\rho(x, y)} \min _{B(y, 1)} g(z, w)
$$

Suppose $\gamma$ has length $\simeq e^{-N}$. Then the boundary piece of $Y$ corresponding to the boundary component $\gamma \subset \partial Y$ has diameter $\simeq N$. Let $A_{n}=\{z \in Y$ : $n-1 \leq \rho(z, \gamma) \leq n\}$. Note that area $\left(A_{n}\right) \leq C e^{n-N}$. Thus

$$
\begin{aligned}
\int_{Y} \exp \left(-4 \rho\left(z, \partial Y_{0}\right)\right) g & (z, w) d A(z) \leq \sum_{n=1}^{N} \int_{A_{n}} e^{-4 n} g(z, w) d A(z) \\
& \leq \sum_{n=1}^{N} e^{-N+n} e^{-4 n} e^{n} \min _{z \in \gamma} g(z, w) \\
& \leq C e^{-N} \min _{z \in \gamma} g(z, w) \\
& \leq C \int_{\gamma} g(z, w) d \sigma(w) .
\end{aligned}
$$




\section{Finding $Y$-pieces near a given point}

In this section, we show there are $L$-bounded $Y$-pieces near any point of $R$.

Lemma 3.1. Suppose $R$ is a Riemann surface such that the injectivity radius is bounded above by $M<\infty$ at every point. Then there is a $L=L(M)<\infty$ so the following holds. Suppose $\gamma$ is a closed geodesic of length $\leq 2 M$ and $\nu$ is a normal direction to $\gamma$. Then there is a generalized $L$-bounded $Y$-piece $Y \subset R$ such that $\gamma$ is one boundary component of $Y$, and $\nu$ points into $Y$.

Proof. Suppose $\gamma$ has hyperbolic length $s$ and lifts to the imaginary axis in the upper half-plane, $\mathbb{H}$. Without loss of generality assume the normal $\nu$ points to the right. The group element corresponding to $\gamma$ is $g(z)=e^{s} z$. Consider the segments

$$
\gamma_{\theta}=\left[e^{i\left(\frac{\pi}{2}-\theta\right)}, e^{s+i\left(\frac{\pi}{2}-\theta\right)}\right) \quad \text { for } \theta \in\left[0, \frac{\pi}{2}\right) .
$$

Clearly $\gamma_{0}$ projects to $\gamma$ in $R$ and each $\gamma_{\theta}$ projects to a closed curve $\tilde{\gamma}_{\theta}$. As $\theta$ increases these sweep out a closed region $W_{\theta}$ in $\mathbb{H}$ which contains larger and larger balls. Since $R$ has injectivity radius bounded by $M$, there is a smallest $\theta_{0}$ (depending only on $M$ ) so the projection of $W_{\theta}$ is no longer 1 to 1 . It is easy to check that $\tilde{\gamma}_{\theta_{0}}$ is not a simple curve (if an interior point of $W_{\theta}$ is identified with some other point of $W_{\theta}$, this must have also been true for a smaller $\theta$ ). Thus we can split $\tilde{\gamma}_{\theta_{0}}$ into two closed curves each with length bounded depending only on $M$. Shrinking each of these to a closed geodesic (or puncture) gives the desired $Y$-piece.

The same proof, with only minor changes, also shows the following.

Lemma 3.2. Suppose $R$ is a Riemann surface such that the injectivity radius is bounded above by $M<\infty$ at every point. Then there is a $L=L(M)<\infty$ so the following holds. Suppose $P$ is a parabolic thin part of $R$. Then there is a L-bounded $Y$-piece $Y \subset R$ such that $P \subset Y$.

The last two lemmas imply that every point of $R$ is near a $L$-bounded $Y$-piece in the following sense.

Lemma 3.3. Suppose $R$ is a Riemann surface such that the injectivity radius is bounded above by $M<\infty$ at every point. Then there is a $L=L(M)<\infty$ and $C=C(M)<\infty$ so that for every point $z \in R$ there is a L-bounded $Y$-piece $Y \subset R$ such that either $z \in Y$ or the distance from $z$ to the thick part of $Y$ is $\leq C$. 
Proof. First suppose $z$ is in a parabolic thin part of $R$. Then by Lemma 3.2 there is a $Y$-piece with the desired estimates containing $z$.

Next suppose $z$ is in a hyperbolic thin part $P$ and let $\gamma$ be the central simple closed geodesic of the part. Then $\gamma$ cuts $P$ into two pieces, one of which contains $z$. Now apply Lemma 3.1 with $\nu$ pointing into the piece containing $z$ to obtain a $Y$-piece containing $z$.

Finally, assume $z$ is in the thick part of $R$. Then there is a simple closed loop $\tilde{\gamma}$ passing through $z$ and of length $\leq 2 M$.

Let $\gamma$ be the simple closed geodesic or puncture corresponding to $\tilde{\gamma}$ and note that $z$ is either a bounded distance from $\gamma$ or from the thin part corresponding to $\gamma$. Using either Lemma 3.1 or 3.2 we get a $Y$-piece whose thick part is only a bounded distance from $z$ (and the bound depends only on $M$ ).

Recall that if $\Omega$ is a domain, $z \in \Omega$ and $E \subset \partial \Omega$, then $\omega(z, E, \Omega)$ denotes the harmonic measure of $E$ in $\Omega$ with respect to $z$. For a fixed $E$ this is a harmonic function on $\Omega$, and for a fixed $z$ is a probability measure on $\partial \Omega$.

Lemma 3.4. Suppose $Y$ is a L-bounded $Y$-piece. Then given $r>0$ there is $a \delta_{1}=\delta_{1}(r, L)$ and a boundary geodesic $\gamma \subset \partial Y$ such that $\omega(z, \gamma, R \backslash \gamma) \geq \delta_{1}$ for every $z \in R$ such that $\rho(z, Y) \leq r$.

Proof. First note that a Brownian motion starting at $z$ has a probability of hitting $Y$ which is bigger than $\delta(r)>0$, depending only on $r$ (it is bounded below by the probability of hitting a unit hyperbolic ball at distance $r+1$ in the hyperbolic disk).

Second, a Brownian motion starting in $Y$ has probability at least a $1 / 2$ of reaching the thick part of $Y$ (since starting on short geodesic it has equal chance of exiting the thin part in either of the two boundary components and if it starts in the thin part in $Y$, then it has a $\geq 1 / 2$ chance of exiting the thin part via the thick part of $Y$ ). Since the diameter of the thick part of $Y$ is bounded, if we fix a boundary component of $Y$ its harmonic measure will be comparable at any two points of the thick part (by Harnack's inequality), say with a constant $C=C(L)$.

Finally, fix a point of the thick part and note that one of the three boundary components of $Y$ must have harmonic measure at least $1 / 3$ with respect to this point. Thus for our original point $z$ the probability of hitting this boundary curve is at least

$$
\delta_{1}=\delta(r) \frac{1}{2} \frac{1}{C} \frac{1}{3} .
$$




\section{Finding disjoint $Y$-pieces}

In this section we will show that given any point $z$ of $R$ we can find a disjoint collection of $X$-pieces which are far from $z$, but have large harmonic measure with respect to $z$.

Lemma 4.1. Suppose $R$ has a Green's function and has injectivity radius bounded by $M, z_{0} \in R$ and $K \subset R$ is compact. Then there is an $\delta_{2}=$ $\delta_{2}(M)>0$ and a finite disjoint collection of simple closed geodesics $\left\{\gamma_{n}\right\}$ of length $\leq L=L(M)$ (the constant from Lemma 3.3) so that

$$
\sum_{n} \omega\left(z_{0}, \cup_{n} \gamma_{n}, R \backslash \cup_{n} \gamma_{n}\right) \geq \delta_{2}
$$

Moreover, if $Y_{n}$ is a L-bounded $Y$-piece with $\gamma_{n}$ as a boundary curve, then $Y_{n} \cap K=\emptyset$. Also, given any $\epsilon>0$ we may choose the curves $\gamma_{n}$ so that the Green's function $g$ with pole at $z_{0}$ is less than $\epsilon$ on every $\gamma_{n}$.

Proof. Fix $s>0$ so large that $K \subset B\left(z_{0}, s\right)$ and consider all hyperbolic thin parts that hit $B\left(z_{0}, s+D(L)+1\right)$ (where $D(L)$ is the bound on the diameter of the thick part of a $L$-bounded $Y$-piece). This is a finite collection so the union is compact, call it $K_{1}$. Now take a $D(L)+1$ neighborhood of $K_{1}$, call it $K_{2}$. Then take all the hyperbolic thin parts which hit it and let the union be $K_{3}$. This is compact, so there is a ball $B\left(z_{0}, t\right)$ which contains it. We claim that if $\gamma$ is a simple closed geodesic which is outside $B\left(z_{0}, t\right)$ then any $L$-bounded $Y$-piece, of which it is a boundary component, is disjoint from $K$.

To prove this claim, note that if $Y$ hits $K$ in its thick part, then it is contained in $K_{1}$. If it hits $K$ with a thin part, then the thick part of $Y$ is contained in $K_{2}$ and so $Y$ is contained in $K_{3}$. Thus $\gamma$ must be in $K_{3}$, contrary to assumption. This proves the claim.

Now choose $\epsilon>0$ so small that $E=\left\{z: g\left(z, z_{0}\right)=\epsilon\right\}$ is outside $B\left(z_{0}, t\right)$ (recall $g$ is the Green's function with pole at $z_{0}$ ). We can do this because $g$ is strictly positive on any compact set. Clearly $E$ has harmonic measure 1 with respect to $z_{0}$.

For each $z \in E$ let $Y_{z}$ be the $Y$-piece associated to $z$ by Lemma 3.3. By Lemma 3.4 there is a geodesic boundary component $\gamma=\gamma_{z}$ which has harmonic measure at least $\delta_{1}$ with respect to $z$. Let $\left\{\gamma_{n}\right\}$ be an enumeration of the geodesics which arise in this way (we will show it is a finite collection in Lemma 4.2). Then clearly,

$$
\omega\left(z_{0}, \cup_{n} \gamma_{n}, R \backslash \cup_{n} \gamma_{n}\right) \geq \delta_{1} \omega\left(z_{0}, E, R \backslash E\right)=\delta_{1},
$$

as desired. 
Finally, since each $\gamma_{n}$ has uniformly bounded length and is far from the pole of Green's function, the value of $g$ at any two points of $\gamma_{n}$ are comparable with uniform constants (depending only on $M$ ). Since $\gamma_{n}$ has harmonic measure $\geq \delta_{1}$ with respect to a point $z$ where $g\left(z, z_{0}\right)=\epsilon$, we deduce that $g$ is bounded by $C \epsilon / \delta_{1}$ on $\gamma_{n}$ (where $C$ depends only on $M$ ). Taking $\epsilon$ even smaller if necessary proves the final claim of the lemma.

Lemma 4.2. Given $L<\infty$ and a compact set $K$, there is an $N_{1}=N(L, K)$ such that $K$ can intersect at most $N_{1}$ different simple closed geodesics of length $\leq L$.

Proof. By the Collar lemma (e.g., [10], [11]), each simple closed geodesic $\gamma$ of length $\leq L$ has a $\delta=\delta(L)$ annular neighborhood $A$ with the property that $\gamma$ is the only simple closed geodesic contained entirely in $A$. Let $\epsilon>0$ and suppose that $K$ can be covered by $n_{1}$ balls of size $\epsilon$. Let $B$ be one of these balls and suppose more than $2 \pi n_{2}+1$ simple closed geodesics hit $B$. Choose one such, say $\gamma_{0}$. Then either there is a geodesic $\gamma_{1}$ hitting $B$ which is disjoint from $\gamma_{0}$ or there are two which both hit $\gamma_{0}$ and the angles they make with $\gamma_{0}$ differ by less than $1 / n_{2}$. In the first case, since both $\gamma_{0}$ and $\gamma_{1}$ have length $\leq L$, the two geodesics must remain within distance $\epsilon+\epsilon e^{L}$ of each other. So if $\epsilon$ is small enough (depending on $L$ and $\delta$ ), they lie in each others $\delta$ collars and hence are the same geodesic.

A similar argument works if there are two geodesics which both hit $\gamma_{0}$ within $\epsilon$ of each other and with angles differing by $1 / n_{2}$. Thus there must be fewer than $n_{2}$ simple closed geodesics of length $\leq L$ hitting $B$, and hence fewer than $n_{1} n_{2}$ which hit $K$.

Lemma 4.3. Suppose $K \subset R$ is a set of diameter at most $D$. Then the number of distinct thin parts which can intersect $K$ is bounded by $N_{2}=$ $N_{2}(D)$.

Proof. Without loss of generality we may assume $K$ is connected (since we can make it connected without increasing its diameter). If $K$ is contained in a single thin part, then we may take $N_{3}=1$. Otherwise, if $K$ intersects a part, it also intersects a component of the part's boundary. Then such a boundary component is also a boundary of an annulus inside the thin part of at most unit width and a fixed hyperbolic area. Thus for each thin part $K$ intersects, the 1-neighborhood of $K$ intersected with the part contains a set of fixed area. Since this neighborhood has diameter $\leq D+2$ its hyperbolic area is bounded in terms of $D$ and hence $K$ can only intersect a bounded number of thin parts. 
Lemma 4.4. Suppose $\gamma$ is a simple closed geodesic of length $\leq L$. Then $\gamma$ can be a boundary component of at most $N_{3}=N_{3}(L)$ distinct L-bounded $Y$-pieces.

Proof. First suppose $\gamma$ is in the thick part of $R$ and $\gamma$ is a boundary component of $Y$. If $\gamma_{1}$ is one of the other two boundary components (either a geodesic or puncture), then either it hits a $D(L)$ neighborhood of $\gamma$ (if it is also in the thick part) or its associated thin part hits the $D(L)$-neighborhood of $\gamma$. In either case, the number of possible such geodesics is bounded in terms of $L$ by Lemma 4.2 and Lemma 4.3.

If $\gamma$ is in a thin part (or is a puncture), then a similar argument works except that we have to consider $D(L)$ neighborhoods of the two boundary components of the thin part associated to $\gamma$.

We will say an $X$-piece is $L$ bounded if it is the union of two $L$-bounded $Y$-pieces.

Lemma 4.5. There is a $N=N(L)$ such that a L-bounded $X$-piece can intersect at most $N$ distinct $L$-bounded $X$-pieces.

Proof. Since a $X$-piece is a union of two $Y$-pieces, it clearly suffices to prove the result for $Y$-pieces instead. So fix a $L$-bounded $Y$-piece $Y_{0}$ and suppose $Y_{1}$ is another $L$-bounded $Y$-piece which intersects it. Since one $Y$ piece cannot properly contain another, a boundary component of one must intersect a boundary component of the other. By Lemma 4.2 each boundary component of $Y_{0}$ can intersect at most $N_{1}$ distinct simple closed geodesics of length $\leq L$. Moreover, each such geodesic can be a boundary component of at most $N_{3}$ distinct $Y$-pieces. Thus $Y_{0}$ can intersect at most $3 N_{1} N_{3}$ distinct $Y$-pieces.

Lemma 4.6. Suppose $R$ has injectivity radius bounded by $M, z_{0} \in R$ and $K \subset R$ is compact. Then there is an $\delta_{3}=\delta_{3}(M)$ and a finite disjoint collection of simple closed geodesics $\left\{\gamma_{n}\right\}$ of length $\leq L=L(M)$ (the constant from Lemma 3.3) so that

$$
\sum_{n} \omega\left(z_{0}, \cup_{n} \gamma_{n}, R \backslash \cup_{n} \gamma_{n}\right) \geq \delta_{3}
$$

Moreover, each $\gamma_{n}$ is contained in an L-bounded $X$-piece $X_{n}$ such that that the collection $\left\{X_{n}\right\}$ is pairwise disjoint and all are disjoint from $K$. Also, given any $\epsilon>0$ we may choose the curves $\gamma_{n}$ so that the Green's function $g$ with pole at $z_{0}$ is less than $\epsilon$ on every $\gamma_{n}$. 
Proof. This is immediate from Lemma 4.1, except for the disjointness of the $X_{n}$ 's. Take the collection of $X$-pieces given by Lemma 4.1 and order then in decreasing order, according to the harmonic measure $\gamma\left(z_{0}, \gamma_{n}, R \backslash \cup_{n} \gamma_{n}\right)$. Form a disjoint subcollection inductively as follows. Take the first $X$-piece (i.e., the one with geodesic of largest harmonic measure) and remove from the list all later $X$-pieces which hit it. Then choose the next remaining element and remove the $X$-pieces which hit it. Continue in this way. The resulting collection is obviously pairwise disjoint and since at each stage we are removing at most $N$ (as in Lemma 4.5) geodesics each of whose harmonic measure is less than a geodesic we keep, we see that the harmonic measure of the remaining geodesics is at least $\delta_{3}=\delta_{2} /(N+1)$. This completes the proof.

If Green's function $g$ is $\leq \epsilon$ on the boundary curve $\gamma$ of an $Y$-piece, then $g$ is bounded by $C \epsilon$ on the thin part corresponding to $\gamma$ (since each boundary component of the thin part has equal harmonic measure from $\gamma$ ) and is also bounded on the central piece of $Y$ (by Harnack's inequality). Thus if $g$ is bounded by $\epsilon$ on the central curve of a $X$-piece, then it is bounded by $C \epsilon$ on the core of $X$.

\section{Defining $\mu$ by potential theory}

In this section we define the desired Beltrami coefficient $\mu$. We will write $\mu=\sum_{n} \mu_{n}$ where $\mu_{n}=\sum_{j} \mu_{j, n}$ and each $\mu_{j, n}$ is a Beltrami coefficient supported on a $X$-piece $X_{j, n}$ which shrinks a simple closed geodesic $\gamma_{j, n} \subset$ $X_{j, n}$ by a factor of $1-\epsilon_{j, n}$. The delicate part is that given $\epsilon>0$ we want to choose the $\epsilon_{j, n}$ 's so that the resulting Beltrami coefficients $\left\{\mu_{n}\right\}$ correspond to Carleson measures $\left|\mu_{n}(z)\right|^{2}(1-|z|)^{-1} d x d y$, with norms $\simeq \epsilon$. We will make this selection using potential theory as our guide.

Suppose we are given a dense sequence $\left\{z_{n}\right\} \subset R$ in which each elements appears infinitely often and an increasing sequence $\left\{r_{n}\right\} \subset[0, \infty)$. Define a collection of $X$-pieces $\left\{X_{j, 1}\right\}$ with central curves $\left\{\gamma_{j, 1}\right\}$ by applying Lemma 4.6 to the point $z_{1}$ and the compact set $K_{1}=\left\{z: \rho\left(z, z_{1}\right) \leq 1\right\} \subset R$. In general, if we have already defined a collection $\left\{X_{j, n-1}\right\}$, let $K_{n}$ be a closed $r_{n}$-neighborhood of $\cup_{k<n} \cup_{j} X_{j, k}$, and let $\delta_{n}=\min _{E_{n}} g\left(z, z_{1}\right)$ (where $g$ denotes the Green's function). Choose $\epsilon_{n}<\delta_{n} / n$. Now define the collection of $X$-pieces $\left\{X_{j, n}\right\}$ and geodesics $\left\{\gamma_{j, n}\right\}$ by applying Lemma 4.6. Thus there is a uniform constant $A$ (depending only on $M$ ) and a sequence $\epsilon_{n} \rightarrow 0$, so that if $X_{n}$ is the union of the cores of $n$th generation $X$-pieces and $S_{n}=\left\{z: \epsilon_{n} / A \leq g\left(z, z_{1}\right) \epsilon_{n}\right\}, S=\cup_{n} S_{n}$, then $\operatorname{dist}\left(X_{n}, S\right) \geq r_{n}$. In other words, distinct generations are well separated by level curves of Green's function. 
Now fix a $n$ and let $\Gamma_{n}=\cup_{j} \gamma_{j, n}$ where $\gamma_{j, n}$ is the simple closed geodesic contained in $X_{j, n}$ and given by Lemma 4.6. Let

$$
u_{n}(z)=\omega\left(z, \Gamma_{n}, R \backslash \Gamma_{n}\right)
$$

This is a superharmonic function on $R$, is harmonic off $\Gamma_{n}$, takes values between 0 and 1 and has boundary values 1 on $\Gamma_{n}$. In the sense of distributions, the Laplacian $\Delta u_{n}$ exists as a measure and is supported on $\Gamma_{n}$. Let $\nu_{n}$ denote this measure on $\Gamma_{n}$. Then

$$
u_{n}(z)=\int_{\Gamma_{n}} g(z, w) d \nu_{n}(w)
$$

where $g$ denotes the Green's function on $R$. Since each geodesic $\gamma_{j, n}$ has uniformly bounded length, there is a fixed width neighborhood $A_{j, n}$ of the geodesic so that $u_{n}$ is harmonic in $A_{j, n} \backslash \gamma_{j, n}$. Since $u_{n}$ is bounded between 0 and 1 , this implies it has uniformly bounded normal derivatives. Since each geodesic has uniformly bounded length, this means the mass $\nu_{n}\left(\gamma_{j, n}\right)$ is uniformly bounded for all $j$ and $n$.

Let $\epsilon_{j, n}=\sqrt{\epsilon\left|\nu_{n}\left(\gamma_{j, n}\right)\right|}$ and let $\mu_{j, n}$ be the Beltrami coefficient of the quasiconformal map given by Lemma 1.2 which is supported in $X_{j, n}$ and shrinks $\gamma_{j, n}$ by a factor of $1-\epsilon_{j, n}$ (by our comments above $\epsilon_{j, n} \leq 1 / 2$ if $\epsilon$ is small enough). Now let

$$
\mu_{n}=\sum_{j} \mu_{j, n} \quad \text { and } \quad \mu=\sum_{n} \mu_{n}
$$

As usual, given an arc $I \subset \mathbb{T}$ we define the corresponding Carleson box as

$$
Q_{I}=\{z \in \mathbb{D}: z /|z| \in I, 1-|z| \leq|I|\} .
$$

The top of the box $Q$ is defined as

$$
T(Q)=\left\{z \in \mathbb{D}: z /|z| \in I, \frac{1}{2}|I| \leq 1-|z| \leq|I|\right\} .
$$

The side length of a box is defined as $\ell(Q)=|I|$. The dyadic subintervals of $\mathbb{T}$ are those of the form

$$
\left[\exp \left(i j 2^{-n}\right), \exp \left(i(j+1) 2^{-n}\right)\right]
$$

and a dyadic Carleson box is one with a dyadic base. A positive measure on disk is Carleson iff $\nu(Q) \leq C \ell(Q)$ for every Carleson box. 
Lemma 5.1. Suppose $\mu_{n}$ is as above. Then $\left|\mu_{n}(z)\right|^{2}\left(1-|z|^{2}\right)^{-1} d x d y$ is a Carleson measure on the disk with norm at most $C \epsilon$ (where $C$ is independent of $n$ and $\epsilon$, but depends on $R$ ).

Proof. The key observation is the following simple result (see e.g., [4]).

Lemma 5.2. Suppose $f$ is a positive function on the disk, bounded by $A<\infty$ and such that for every $w \in \mathbb{D}$,

$$
\int_{\mathbb{D}} f(z) \log \left|\frac{1-\bar{w} z}{z-w}\right| \frac{d x d y}{\left(1-|z|^{2}\right)^{2}} \leq A<\infty .
$$

Then $f(z)\left(1-|z|^{2}\right)^{-1} d x d y$ is a Carleson measure with norm at most $C A$.

In this lemma, the function multiplying $f$ is the Green's function for the disk and $d x d y /\left(1-|z|^{2}\right)^{2}$ is hyperbolic area on the disk. Thus if $f$ is invariant under a Fuchsian group $G$, it is a well defined function on $R$ and the integral in the lemma becomes

$$
\int_{R} f(z) g(z, w) d A(z)
$$

where $g$ is the Green's function for $w$ and $d A$ denotes hyperbolic area on $R=\mathbb{D} / G$. Thus to prove Lemma 5.1 , we need only show

$$
\sup _{w \in R} \int_{R}\left|\mu_{n}(z)\right|^{2} g(z, w) d A(z) \leq C \epsilon .
$$

By Lemma 2.4,

$$
\int_{R}\left|\mu_{n}(z)\right|^{2} g(z, w) d A(w) \leq C \epsilon \sum_{j} \int_{\gamma_{j, n}} g(z, w) d \nu_{n}(w)=C \epsilon u_{n}(z) \leq C \epsilon .
$$

This completes the proof of Lemma 5.1

Choose an $a>0$ so that the components of $\Gamma$ are at least hyperbolic $a$ distance apart (we can do this by the collar lemma since all the components are closed geodesic of length $\leq L)$.

Lemma 5.3. Let $\tilde{\Gamma}_{n}$ be the lift of $\Gamma_{n}$ to the unit disk and let $W$ be a hyperbolic a-neighborhood of $\tilde{\Gamma}$. On each component of $W$ let $\sigma_{n}(z)$ be $\left|\nu_{n}\left(\gamma_{j, n}\right)\right| / \ell\left(\gamma_{j, n}\right)$ where $j$ is chosen so the component of $W$ projects onto a neighborhood of $\gamma_{j, n}$. Then for any Carleson square $Q \subset \mathbb{D}$,

$$
\limsup _{n} \int_{Q \cap W} \sigma_{n}(z) \frac{d x d y}{1-|z|^{2}} \geq \delta \ell(Q)>0
$$

where $\delta>0$ depends only on $M$, the injectivity bound for $R$. 
Proof. Fix a Carleson square $Q=Q_{I}$ and for $0<\lambda<1$ let $\lambda Q$ denote the Carleson square whose base is centered at the same point that $I$ is, but whose length is $\lambda|I|$. We will show the desired estimate holds for $Q$ if $\lambda$ is small enough and $z_{n} \in T(\lambda Q)$. Since $\left\{z_{n}\right\}$ is dense in $R$, this implies the lemma. Note that

$$
\ell(Q)^{-1} \int_{Q \cap W} \sigma_{n}(z) \frac{d x d y}{1-|z|^{2}} \geq \int_{Q \cap W} \sigma_{n}(z) \tilde{g}\left(z, z_{n}\right) d A(z),
$$

where $\tilde{g}$ is the Green's function for the disk and $d A$ denotes hyperbolic area. Thus

$$
\begin{aligned}
\int_{Q \cap W} \sigma_{n}(z) \frac{d x d y}{1-|z|^{2}} \geq \int_{W} & \sigma_{n}(z) \tilde{g}\left(z, z_{n}\right) d A(z) \\
& -\int_{Q^{c} \cap W} \sigma_{n}(z) \tilde{g}\left(z, z_{n}\right) d A(z) .
\end{aligned}
$$

The first integral on the right is the same as

$$
\int_{R} \sigma_{n}(z) g\left(z, z_{n}\right) d A(z) \geq C \int_{R} g\left(z, z_{n}\right) d \nu_{n} \geq C u\left(z_{n}\right) \geq C_{1} \delta_{3} .
$$

To estimate the second integral on the right side of (5.1) use a Möbius transformation to move $z_{n}$ to the origin. Then $Q^{c}$ is mapped into a Carleson square $Q^{\prime}$ of diameter $\simeq \lambda$ and so

$$
\begin{aligned}
\int_{Q^{c} \cap W} \sigma_{n}(z) \tilde{g}\left(z, z_{n}\right) d A(z) & \leq \int_{Q^{\prime}} \sigma_{n}(z) \log \frac{1}{|z|} d A(z) \\
& \leq \int_{Q^{\prime}} \sigma_{n}(z) \frac{d x d y}{1-|z|^{2}} \\
& \leq C_{2} \lambda
\end{aligned}
$$

where the last inequality holds because the proof of Lemma 5.1 shows that $\sigma_{n}(z)\left(1-|z|^{2}\right)^{-1} d x d y$ is a Carleson measure with uniformly bounded norm. Thus if we choose $\lambda<C_{1} \delta_{3} /\left(2 C_{2}\right)$, we get

$$
\ell(Q)^{-1} \int_{Q \cap W} \sigma_{n}(z) \frac{d x d y}{1-|z|^{2}} \geq \delta_{3} / 2
$$

which proves the lemma.

The following is now immediate.

Corollary 5.4. The Carleson norm of $\left|\mu_{n}(z)^{2}\right|\left(1-|z|^{2}\right)^{-1} d x d y$ is comparable to $\epsilon$ with constants that only depend on the injectivity upper bound for $R$. 


\section{Divergence of a Stolz type sum}

Suppose $\nu$ is a Carleson measure on the $\mathbb{D}$ with constant $M$ and for $x \in \mathbb{T}$, let

$$
I_{\nu}(x)=\sum_{I} \nu\left(T\left(Q_{I}\right)\right)|I|^{-1}
$$

where the sum is over all dyadic intervals containing $x$. This is a dyadic analog of an integral over a Stolz cone. It is easy to see that

$$
\int_{\mathbb{T}} I_{\nu}(x) d x=\nu(\mathbb{D})
$$

so that $I_{\nu}$ must be positive on a set of positive measure (if $\nu$ is non-trivial). We need a more quantified version of this fact.

Lemma 6.1. Suppose $\nu$ is a Carleson measure on $\mathbb{D}$ with constant $\leq M$ and suppose $Q$ is a dyadic Carleson square with base $I \subset \mathbb{T}$ such that $\nu(Q) \geq$ $\epsilon \ell(Q)$ for some $\epsilon>0$. Then there is a $B=B(M, \epsilon)$ such that

$$
\left|\left\{x \in I: I_{\nu}(x)>\epsilon / 4\right\}\right| \geq B \nu\left(Q_{I}\right) .
$$

Proof. Without loss of generality, we may assume $\nu$ is supported in $Q$. The idea is to show that $I_{\nu}$ is in dyadic BMO and then apply the John-Nirenberg theorem. To be more precise, recall that a function $f$ on the circle is said to be in dyadic BMO if for every dyadic interval $I$ there is a number $c_{I}$ such that

$$
\|f\|_{\mathrm{BMO}}=\sup _{I} \frac{1}{|I|} \int_{I}\left|f-c_{I}\right|<\infty
$$

and the supremum is over all dyadic intervals. If $f$ is in dyadic BMO then the John-Nirenberg theorem (e.g., [7]), states that there is a $C_{1}, C_{2}<\infty$ such that

$$
\left|\left\{x \in I:\left|f-m_{I}(f)\right| \geq \lambda\right\}\right| \leq C_{1}|I| \exp \left(-C_{2}\|f\|_{\mathrm{BMO}} \lambda\right) .
$$

We claim that if $\nu$ is a Carleson measure then $I_{\nu}$ is in dyadic BMO. To prove this let $c_{I}=\sum_{J} \nu(T(J))$ where the sum is over all dyadic $J$ containing $I$. Then

$$
\frac{1}{|I|} \int\left|I_{\nu}-c_{I}\right| d x \leq\|\nu\|_{C} .
$$


Thus $I_{\nu}$ is in dyadic BMO, as claimed. Hence if $f=I_{\nu}-c_{I}$,

$$
\begin{aligned}
\nu\left(Q_{I}\right) & \leq \int_{I} f d x \\
& \leq \int_{f<\epsilon / 4}+\int_{\epsilon / 4 \leq f<\lambda}+\int_{f \geq \lambda} \\
& \leq \frac{\epsilon}{4}|I|+\lambda\left|\left\{\frac{\epsilon}{4} \leq f<\lambda\right\}\right|+C_{1}|I| \int_{\lambda}^{\infty} \lambda e^{-C_{2} \lambda} d \lambda \\
& \leq \frac{\epsilon}{4}|I|+\lambda\left|\left\{\frac{\epsilon}{4} \leq f<\lambda\right\}\right|+C_{1}|I| e^{-C_{3} \lambda} .
\end{aligned}
$$

Thus taking $\lambda$ so large that $C_{1} e^{-C_{3} \lambda} \leq \epsilon / 4$ we get

$$
\begin{aligned}
\left|\left\{x \in I: I_{\nu} \geq \epsilon / 4\right\}\right| & \geq \frac{1}{\lambda}\left[\nu\left(Q_{I}\right)-\frac{\epsilon}{4}|I|-C_{1}|I| e^{-C_{3} \lambda}\right] \\
& \geq \frac{1}{\lambda}\left[\epsilon|I|\left(1-\frac{1}{4}-\frac{1}{4}\right)\right] \\
& \geq \frac{\epsilon}{2 \lambda}|I| .
\end{aligned}
$$

This completes the proof of the lemma.

Lemma 6.2. Suppose $\left\{\nu_{n}\right\}$ are positive Carleson measures on $\mathbb{D}$, that

$$
\sup _{n}\left\|\nu_{n}\right\|_{C} \leq M<\infty
$$

and that for any Carleson square $Q$,

$$
\limsup _{n} \nu_{n}(Q)=\epsilon>0
$$

(for some $\epsilon$ independent of $Q$ ). Let $\nu=\sum_{n} \nu_{n}$. Then $I_{\nu}(x)=\infty$ almost everywhere on $\mathbb{T}$.

Proof. Fix an $I$. By Lemma 6.1 we have $I_{\nu_{n}}>\epsilon / 8$ on a set of size $B|I|$ for infinitely many $n$ 's. Thus the series $I_{\nu}=\sum_{n} I_{\nu_{n}}$ diverges on a subset of $I$ of size at least $\delta=B|I|$. Since the series diverges on a fixed fraction of every dyadic interval, the Lebesgue density theorem implies it diverges almost everywhere. 


\section{How close is $\Gamma$ to a circle?}

In order to show that $\Gamma=f_{\mu}(\mathbb{T})$ has tangents almost nowhere, we have to show that $\Gamma$ has oscillation at almost all points and infinitely many scales. To do this we measure its oscillations using Peter Jones' $\beta$ 's, e.g., [9].

Given a closed curve $\Gamma$, a point $x \in \Gamma$ and $t>0$ we define

$$
\beta_{\Gamma}(x, t)=\inf _{L \in \mathcal{L}} \frac{1}{t} \sup _{z \in \Gamma \cap B(x, t)} \operatorname{dist}(z, L),
$$

where $\mathcal{L}$ is the set of all lines hitting $x$. For a Riemann mapping $f: \mathbb{D} \rightarrow \Omega$ let $\beta(z)=\beta_{\partial \Omega}(f(z /|z|), 10 \operatorname{dist}(z, \partial \Omega))$. It follows from the results in [5] that if $\Gamma=\partial \Omega$ is a quasicircle, then up to sets of zero 1-dimensional measure, $x=f\left(e^{i \theta}\right)$ is a tangent point of $\Gamma$ iff

$$
\int_{0}^{1} \beta_{\Gamma}\left(r e^{i \theta}\right)^{2} \frac{d r}{1-r}<\infty
$$

or (equivalently, up to sets of linear measure zero) iff,

$$
\int_{0}^{\infty} \beta_{\Gamma}(x, t)^{2} \frac{d t}{t}<\infty
$$

We also define

$$
\mathfrak{B}_{\Gamma}(z, t)=\sum_{n=0}^{\infty} \beta_{\Gamma}\left(z, e^{n} t\right) e^{-n} .
$$

When $\Gamma$ is clear from context, we will drop it from our notation.

Lemma 7.1. With notation as above,

$$
\int_{0}^{\infty} \beta(z, t)^{2} \frac{d t}{t} \simeq \int_{0}^{\infty} \mathfrak{B}(z, t)^{2} \frac{d t}{t} .
$$

Proof. One direction is obvious since $\beta \leq \mathfrak{B}$. To prove the other direction we use Cauchy-Schwarz to get

$$
\begin{aligned}
\int_{0}^{\infty} \mathfrak{B}(z, t)^{2} \frac{d t}{t} & =\int_{0}^{\infty}\left(\sum_{n=0}^{\infty} \beta\left(z, e^{n} t\right) e^{-n}\right)^{2} \frac{d t}{t} \\
& \leq \int_{0}^{\infty}\left(\sum_{n=0}^{\infty} \beta^{2}\left(z, e^{n} t\right) e^{-n}\right)\left(\sum_{n=0}^{\infty} e^{-n}\right) \frac{d t}{t} \\
& =C \sum_{n=0}^{\infty} \int_{0}^{\infty} \beta^{2}\left(z, e^{n} t\right) e^{-n} \frac{d t}{t} \\
& =C \sum_{n=0}^{\infty}\left[\int_{0}^{\infty} \beta^{2}(z, s) \frac{d s}{s}\right] e^{-n}=C^{2} \int_{0}^{\infty} \beta^{2}(z, s) \frac{d s}{s}
\end{aligned}
$$


For our purposes in this paper, it is more convenient to measure the flatness of $\Gamma$ is a slightly different way. We define

$$
\eta_{\Gamma}(z)=\min \left(1, \inf _{\tau} \sup _{x, y \in \Gamma} \log \frac{|\tau(x)|}{|\tau(y)|}\right)
$$

where the infimum is over all Möbius transformations $\tau$ such that $\tau(z)=0$. In other words, $\eta$ measures the smallest $r$ such that $\Gamma$ can be mapped into the annulus $\{1 \leq|z| \leq r\}$ with $z$ going to the origin. The minimum with 1 is included simply to make it easier to compare $\eta$ to $\mathfrak{B}$ below. The main advantage of $\eta$ over $\beta$ is that $\eta$ is invariant under Möbius transformations. In particular, if $\Gamma$ is the limit set of a Kleinian group then $\eta$ is well defined on the quotient Riemann surface. In fact, we may consider $\eta$ as a geometric analog of the Schwarzian derivative.

Lemma 7.2. There is a $C<\infty$ such that the following holds. If $\Gamma \subset \mathbb{R}^{2}$, $z \in \Gamma, t>0$, and $t \leq|w-z| \leq 2 \operatorname{dist}(w, \Gamma) \leq 4 t$ then

$$
\eta_{\Gamma}(w) \leq C \mathfrak{B}_{\Gamma}(z, t)
$$

Proof. After a linear change of variables we may assume that $w=i$ and $-1,1 \in \Gamma$. Let $B_{r}$ be the hyperbolic ball of radius $r$ centered at $i$ in the upper half-plane, $\mathbb{H}$, and let $B_{r}^{*}$ denote its reflection across the real line. Let $\tau$ be a Möbius transformation which maps $\mathbb{H}$ to the unit circle, taking $i$ to 0 . Suppose that $0<a<1$ and that $\mathfrak{B}_{\Gamma}(0,1) \leq a e^{-n}$. We claim that if $a$ is small enough (independent of $n$ ), then $\Gamma \cap\left(B_{n-c} \cup B_{n-c}^{*}\right)=\emptyset$ for some $c>0$ independent of $n$. If we can prove the claim then $\tau(\Gamma)$ lies in a $C e^{-n}$ neighborhood of the unit circle and we will be done.

Thus it suffices to prove the claim. Let $S_{0}=\{z=x+i y:|z| \leq 1,|y| \leq$ $\left.e^{-n}\right\}$ and for $k>0$, let $A_{k}=\left\{z: e^{k-1} \leq|z| \leq e^{k}\right\}, C_{k}=\left\{z:|z|=e^{k}\right\}$ and $S_{k}=\left\{z=x+i y \in A_{k}:|y| \leq e^{2 k-n}\right\}$. It is easy to check that the hyperbolic distance from $i$ to $S_{k}$ in $\mathbb{H}$ is greater than $n-c$ for some $c$ independent of $k$, so it suffices to prove $\Gamma \subset \cup_{k} S_{k}$.

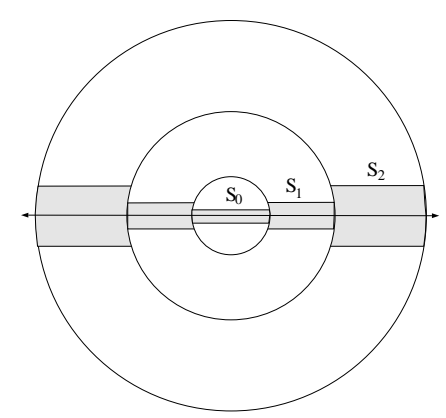

Figure 1: Definition of the $S_{k}$ 
We prove this by induction on $k$. Since $\beta \leq \mathfrak{B}$, we clearly have $\Gamma \cap\{|z| \leq$ $1\} \subset S_{0}$. Now suppose $\Gamma \cap A_{k-1} \subset S_{k-1}$. Since $\mathfrak{B}_{\Gamma}(0) \leq a e^{-n}$ we must have

$$
\beta_{k} \equiv \beta_{\Gamma}\left(i, e^{k}\right) \leq e^{k-n} \frac{e-1}{e(e+1)}
$$

if $a$ is small enough. Hence $\Gamma \cap A_{k}$ is contained in strip $S$ of width $2 \beta_{k} e^{k}=$ $e^{2 k-n-1}$. Since $\Gamma \cap A_{k-1} \subset S_{k-1}, S \cap S_{k} \cap C_{k-1} \neq \emptyset$. Thus the maximum height of a point in $S \cap C_{k-1}$ is at most $e^{k-n}+\beta_{k} e^{k}$. Thus the central line of $S$ (which passes through the origin) hits $C_{k}$ at height at most $e$ times this and so $S \cap A_{k}$ has height at most

$$
\begin{aligned}
e\left[e^{2(k-1)-n}+\beta_{k} e^{k}\right]+\beta_{k} e^{k} & =e^{2 k-1-n}+\left(e^{2 k-n+1}+e^{2 k-n}\right) \frac{e-1}{e(e+1)} \\
& =e^{2 k-n}\left(e^{-1}+(e+1) \frac{e-1}{e(e+1)}\right)=e^{2 k-n} .
\end{aligned}
$$

Thus $\Gamma \cap A_{k} \subset S_{k}$, as desired. This completes the proof of the lemma.

From the lemma we see that there is a $C<\infty$ so that for any $\Gamma=f(\mathbb{T})$ and any $e^{i \theta} \in \mathbb{T}$

$$
\int_{0}^{1} \eta_{\Gamma}^{2}\left(f\left(r e^{i \theta}\right)\right) \frac{d r}{1-r} \leq C \int_{0}^{1} \mathfrak{B}_{\Gamma}^{2}\left(r e^{i \theta}\right) \frac{d r}{1-r}
$$

Hence, combining (7.1), Lemma 7.1 and Lemma 7.2 we get

Corollary 7.3. Except for a set of zero 1-dimensional measure, $f\left(e^{i \theta}\right)$ is not a tangent point of $\Gamma$ if

$$
\int_{0}^{1} \eta_{\Gamma}\left(f\left(r e^{i \theta}\right)\right)^{2} \frac{d r}{1-r}=\infty
$$

\section{Shrinking a geodesic makes $\eta$ large}

In this section we show that shrinking a closed geodesic forces $\eta$ to be large. Combined with the results of the previous sections, this will imply that $f_{\mu}(\mathbb{T})$ has tangents almost nowhere.

Lemma 8.1. Given $2 \leq M<\infty$, there is a $C=C(M)<\infty$ so that the following is true. Suppose $\Gamma$ is a closed Jordan curve which is the limit set of a Kleinian group $G$. Let $\Omega_{1}$ be the bounded complementary component and $\Omega_{2}$ the unbounded component. Suppose that $g \in G$ fixes $-1,1 \in \Gamma$ and that $g$ has translation length $r \leq M$ in $R_{1}=\Omega_{1} / G$ and length $(1-\epsilon) r$ in $R_{2}=\Omega_{2} / G$. Then $\eta_{\Gamma}(0) \geq C \epsilon$.

Proof. Let $\rho_{j}$ denote hyperbolic distance on $\Omega_{j}$ for $j=1,2$. Let $\rho$ denote the hyperbolic metric on $\mathbb{D}$ and also on $\mathbb{D}^{*}=\{|z|>1\} \cup\{\infty\}$. We may assume that $\eta$ is small, say $\eta<\delta=\delta(M)$ (to be chosen below), because otherwise the lemma is true with $C=1 / \delta$. 
Note that if $\Gamma \subset\left\{\frac{1}{1+\eta} \leq|z| \leq 1+\eta\right\}$, then

$$
\frac{1}{1+C \eta} \leq \frac{d \rho_{1}}{d \rho} \leq 1+C \eta
$$

for $|z| \leq 1-\delta$ or $|z| \geq 1+\delta$ and $C$ depends on $\delta$. The translation length of $g$ in $\Omega_{j}$ for $j=1,2$ is equal to

$$
\min _{z \in \Omega_{j}} \rho_{j}(z, g(z)) \text {. }
$$

By replacing $g$ by a power of itself, we may assume its translation length in $\Omega_{1}$ is at least 1 . If $\delta$ is small enough then the axis $A$ of $g$ from -1 to +1 must intersect $B_{1 / 2}=\{|z| \leq 1 / 2\}$. Choose a point $z \in A \cap B_{1 / 2}$. Since $g$ has translation length $\leq M$ the image $g(z)$ is still in $\{|w| \leq 1-\delta\}$ (if $\delta$ is small enough depending on $M$ ).

The Möbius transformation $g$ can be written as a composition $g=\sigma \circ \tau$ where both $\tau$ and $\sigma$ fix 1 and $-1, \tau$ maps the unit circle to itself (and hence $\left.\tau\left(z^{*}\right)=\tau(z)^{*}\right)$ and $\sigma$ moves points by less than $C \eta$ in the spherical metric. Thus

$$
\begin{aligned}
\rho_{1}(z, g(z)) & \leq(1+C \eta) \rho(z, g(z))=(1+C \eta) \rho\left(z^{*}, g(z)^{*}\right) \\
& \leq(1+C \eta)\left[\rho\left(z^{*}, g\left(z^{*}\right)\right)+\rho\left(g\left(z^{*}\right), g(z)^{*}\right)\right] \\
& \left.\leq(1+C \eta)\left[\rho\left(z^{*}, g\left(z^{*}\right)\right)+\rho\left(\sigma\left(\tau(z)^{*}\right)\right), \sigma(\tau(z))^{*}\right)\right] \\
& \leq(1+C \eta)\left[(1+\eta) \rho_{2}\left(z^{*}, g\left(z^{*}\right)\right)+C \eta\right] \\
& \leq(1+C \eta) \rho_{2}\left(z^{*}, g\left(z^{*}\right)\right) .
\end{aligned}
$$

Hence the translation length in $\Omega_{1}$ is at most $(1+C \eta)$ times the translation length in $\Omega_{2}$ and hence $\epsilon<C \eta$, as desired.

\section{9. $f_{\mu}(\mathbb{T})$ has almost no tangents}

Suppose $\mu=\sum_{n} \mu_{n}$ is the Beltrami coefficient defined in Section 5 and $f_{\mu}$ is the corresponding quasiconformal map. Each $\mu_{n}$ is a sum of the form $\sum \mu_{n, j}$ where each $\mu_{n, j}$ is associated to a closed geodesic $\gamma_{n, j}$ and shrinks the length of this geodesic by a factor of $1-\epsilon_{n, j}$. Let $A_{n, j}$ be a hyperbolic $a$ neighborhood of $\gamma_{n, j}$ (where $a$ is chosen so these neighborhoods are disjoint). Let $\sigma_{n, j}=\epsilon_{n, j}^{2} \chi_{A_{n, j}}, \sigma_{n}=\sum_{j} \sigma_{n, j}$ and $\sigma=\sum_{n} \sigma_{n}$.

Combining Lemmas 5.1, 5.3 and 6.2, we see that $I_{\sigma}=\infty$ almost everywhere on the circle. By Lemma 8.1 we know that $\eta(z)^{2} \geq C \epsilon_{n, j}^{2}$ on $A_{n, j}$. Thus

$$
\int \eta^{2}(r x) \frac{d r}{1-r} \gtrsim I_{\sigma}(x)=\infty
$$

for almost every $x$ on the circle. Thus $f_{\mu}(\mathbb{T})$ has tangents on a set of at most linear measure zero, by Corollary 7.3. 


\section{A stopping time decomposition}

A stopping time region $W \subset \mathbb{D}$, is a region of the form

$$
W=Q \backslash \cup_{k} Q_{k},
$$

where $Q$ and $\left\{Q_{k}\right\}$ are dyadic Carleson squares. For dyadic intervals, $I \cap J \neq$ $\emptyset$ implies $I \subset J$ or $J \subset I$ and from this is easily follows that the $\left\{Q_{k}\right\}$ in the definition of stopping time region may always be taken to have disjoint interiors.

A locally rectifiable curve $\gamma$ is called a chord-arc (or Lavrentiev) curve if there is a $C<\infty$ so that for any two points $x, y \in \gamma$, the shorter arc between $x, y$ has length at most $C|x-y|$. A domain $W$ will be called chord-arc if its boundary is a closed chord-arc curve. Clearly, dyadic stopping-time regions are chord-arc domains (with a uniform constant).

We will be interested in stopping time regions formed in the following way. Let $R=\mathbb{D} / G$ be a Riemann surface with Green's function. Fix a base point $z_{0} \in R$ and let $g\left(z, z_{0}\right)$ denote the lift to the disk of the Green's function on $R$ with pole at $z_{0}$. Note that away from the lifts of $z_{0}, g\left(z, z_{0}\right)$ is positive harmonic function and hence by Harnack's inequality there is a constant $A$ so that

$$
\max _{T(Q)} g\left(z, z_{0}\right) \leq A \min _{T(Q)} g\left(z, z_{0}\right),
$$

for any top half of a Carleson box for which $\max _{T(Q)} g\left(z, z_{0}\right) \leq 1$.

Now suppose $1>\epsilon_{0}>\epsilon_{1}>\ldots$ are as in Section 5 , so that the level sets $\left\{g=\epsilon_{n}\right\}$ separate the supports of the $\mu_{n}$ 's. We may assume that $z_{0}$ is the projection of the origin via the quotient map $\mathbb{D} \rightarrow R$. Let $\mathcal{C}_{1,0}$ be the collection of dyadic Carleson boxes which are maximal with respect to the property

$$
\max _{T(Q)} g\left(z, z_{0}\right) \leq \epsilon_{1}
$$

and let

$$
W_{0}=\mathbb{D} \backslash \cup_{Q \in \mathcal{C}_{0,1}} Q .
$$

Then $W_{0}$ is a chord-arc domain and

$$
\begin{aligned}
g\left(z, z_{0}\right) & \geq \epsilon_{1} / A, \quad z \in W_{0}, \\
\epsilon_{1} \geq g\left(z, z_{0}\right) & \geq \epsilon_{1} / A, \quad z \in \partial W_{0} .
\end{aligned}
$$

For each $Q \in \mathcal{C}_{1,0}$ define the collection $\mathcal{C}(Q)=\mathcal{C}_{2,1} \cup \mathcal{C}_{0,1}$ where $\mathcal{C}_{2,1}$ is the maximal collection of dyadic sub-boxes such that

$$
\max _{T(Q)} g\left(z, z_{0}\right) \leq \epsilon_{2},
$$


and $\mathcal{C}_{0,1}$ is the maximal collection of dyadic sub-boxes such that

$$
\max _{T(Q)} g\left(z, z_{0}\right) \geq \epsilon_{0}
$$

In general, given a $Q \in \mathcal{C}_{k, n}$ with $k \geq 2$, define $\mathcal{C}_{k+1, n+1}$ as the maximal collection of dyadic sub-boxes $Q^{\prime}$ of $Q$ such that

$$
\max _{T\left(Q^{\prime}\right)} g\left(z, z_{0}\right) \leq \epsilon_{k+1}
$$

and define $\mathcal{C}_{k-1, n+1}$ as the maximal collection of sub-boxes such that.

$$
\max _{T\left(Q^{\prime}\right)} g\left(z, z_{0}\right) \geq \epsilon_{k-1}
$$

If $Q \in \mathcal{C}_{k, n}$, define the stopping time region

$$
W(Q)=Q \backslash \cup_{Q^{\prime} \in \mathcal{C}_{k-1, n+1} \cup \mathcal{C}_{k+1, n+1}} Q^{\prime} .
$$

We will say such a $W$ is of type $(k, n)$. Note that

$$
\epsilon_{k+1} / A \leq g\left(z, z_{0}\right) \leq \epsilon_{k-1}, z \in W
$$

Furthermore, $\partial W$ is a chord-arc curve, $\partial W \cap \mathbb{T}$ has length zero (since the Green's function $g$ tends radially to 0 almost everywhere on the circle), and

$$
\epsilon_{k} / A \leq g\left(z, z_{0}\right) \leq \epsilon_{k}
$$

on the unique connected component of $\partial W$ which intersects $T(Q)$. Moreover, on every other connected component of $\partial W \backslash \mathbb{T}$ either

$$
\epsilon_{k-1} / A \leq g\left(z, z_{0}\right) \leq \epsilon_{k-1}
$$

or

$$
\epsilon_{k+1} / A \leq g\left(z, z_{0}\right) \leq \epsilon_{k+1} .
$$

If $k=0$ then we have to modify the construction because there is no $\epsilon_{k-1}$. In this case, we just define one collection $\mathcal{C}_{1, n+1}$ as above and let

$$
W(Q)=Q \backslash \cup_{Q^{\prime} \in \mathcal{C}_{1, n+1}} Q^{\prime},
$$

and say $W$ is type $(0, n)$. As above $\partial W$ hits the circle in a set of measure zero,

$$
\begin{gathered}
\epsilon_{1} / A \leq g\left(z, z_{0}\right), z \in W, \\
\epsilon_{0} / A \leq g\left(z, z_{0}\right) \leq \epsilon_{0},
\end{gathered}
$$


for $z$ in the unique component of $\partial W$ hitting $T(Q)$ and

$$
\epsilon_{1} / A \leq g\left(z, z_{0}\right) \leq \epsilon_{1},
$$

for $z$ in all the other components of $\partial W \backslash \mathbb{T}$.

If $k=N$ then we also modify the construction to prevent there being a type $N+1$ region. In this case, we just define one collection $\mathcal{C}_{N-1, n+1}$ as above and let

$$
W(Q)=Q \backslash \cup_{Q^{\prime} \in \mathcal{C}_{N-1, n+1}} Q^{\prime},
$$

and say $W$ is type $(N, n)$. As above,

$$
g\left(z, z_{0}\right) \leq \epsilon_{N-1}, z \in W,
$$

and

$$
\epsilon_{N} / A \leq g\left(z, z_{0}\right) \leq \epsilon_{N}
$$

for $z$ in the unique component of $\partial W$ hitting $T(Q)$ and

$$
\epsilon_{N-1} / A \leq g\left(z, z_{0}\right) \leq \epsilon_{N-1},
$$

for $z$ in all the other components of $\partial W \backslash \mathbb{T}$. However, unlike the previous domains, $\partial W$ hits the circle in a set of positive measure. Let $\mathcal{C}=\cup_{j, n} \mathcal{C}_{j, n}$.

We can think of the decomposition of the disk into these regions as a random walk on $\{0,1, \ldots N+1\}$ which is reflecting at 0 and absorbing at $N+1$ : a type $k$ region is followed by either a type $k-1$ or type $k+1$ region except that a type 1 must be followed by type 2 and a type $N$ has a positive probability of having no successor. Later we will choose the parameters $\left\{\epsilon_{k}\right\}$ so that with high probability a type $k$ is followed by a $k+1$ and not a $k-1$.

Lemma 10.1. There are $\alpha>0$ and $C<\infty$ so that the following holds. Suppose $Q \in \mathcal{C}_{k, n}$ and $W$ is the corresponding stopping time region constructed above. Suppose $E \subset \partial W$ corresponds to the tops of type $(k-1, n+1)$ squares. Then

$$
\ell(E) \leq C\left(\frac{\epsilon_{k}}{\epsilon_{k-1}}\right)^{\alpha} \ell(\partial W)
$$

Proof. Since the Green's function $g$ is harmonic and satisfies $g\left(z_{Q}\right) \geq \epsilon_{k} / A$ and $g(z) \leq \epsilon_{k-1}$ for $z \in E$, we see that the harmonic measure of $E$ in $W$ with respect to $z_{Q}$ satisfies

$$
\omega\left(z_{Q}, E, W\right) \leq A \frac{\epsilon_{k}}{\epsilon_{k-1}} .
$$

Since harmonic measure and arclength are $A_{\infty}$ related with constants depending only on the chord-arc constant of $W$ (this is a theorem of Jerison and Kenig, e.g., [8]) there is an $\alpha$ and $C$ (depending only on the chord-arc constant of $W)$ such that $\ell(E) \leq C \omega(E)^{\alpha}$. Combined with our previous observation, this proves the lemma. 


\section{Chord-arc domains and Carleson measures}

If $W$ is a chord-arc domain then a positive measure $\nu$ on $\mathbb{R}^{2}$ is called a Carleson measure for $\partial W$ if there is a $C<\infty$ so that

$$
\nu(B(x, r)) \leq C r
$$

for every $x \in \partial W$ and $r>0$. The following is due to Stephen Semmes [13].

Theorem 11.1. Suppose $W$ is a chord-arc domain. There is an $\epsilon_{0}$, depending only on the chord-arc constant $M$ of $W$ so that the following holds. Suppose $\mu \in L^{\infty}\left(\mathbb{R}^{2}\right)$ and

$$
d \nu=\frac{\mu^{2}(z)}{\operatorname{dist}(z, \partial W)} d x d y
$$

is a Carleson measure for $W$ with norm $\leq \epsilon_{0}$. Let $f_{\mu}$ be a quasiconformal mapping with dilatation $\mu$. Then $f_{\mu}(W)$ is chord-arc with constant depending only on $\|\nu\|_{C}$ and $M$.

We want to be able to apply Semmes' result to our Beltrami coefficient $\mu$ and the stopping-time domains in our collection $\mathcal{C}$. To do this we must prove

Lemma 11.2. Given $\epsilon>0$ there is an $r<\infty$ so that the following holds. Let $\Gamma=\cup_{W \in \mathcal{C}} \partial W$ and suppose $f=\sum_{n} f_{n}$ is such that the supports of the $f_{n}$ are pairwise disjoint, $\|f\|_{\infty} \leq \epsilon$ and for all $n$,

$$
\frac{f_{n}}{1-|z|} d x d y
$$

is a Carleson measure for $\mathbb{D}^{*}$ with norm $\leq \epsilon$ and that $\rho\left(\operatorname{supp}(f), \Gamma^{*}\right) \geq r$. Then for any $W \in \mathcal{C}$,

$$
\int_{B(x, t)} \frac{f(z)}{\operatorname{dist}(z, \partial W)} d x d y \leq C \epsilon t
$$

for any $x \in \partial W$ and $t>0$.

Proof. Choose a point $x \in \partial W$ and a $t>0$. If $t \geq 2 \operatorname{diam}(W)$ then

$$
\int_{B(x, t) \backslash B(x, \operatorname{diam}(W))} \frac{f(z)}{\operatorname{dist}(z, \partial W)} d x d y \leq \int_{B(x, t)} \frac{f(z)}{1-|z|} d x d y \leq C \epsilon t .
$$

Thus it suffices to consider $t \leq 2 \operatorname{diam}(W)$. 
Choose a Carleson square $Q$ such that $B(x, t) \cap \mathbb{D} \subset Q$ and $\operatorname{diam}(Q) \simeq t$. Clearly it suffices to show that (recall $Q^{*}$ and $W^{*}$ are the reflections of $Q$ and $W$ across the unit circle)

$$
\int_{Q^{*}} \frac{f(z)}{\operatorname{dist}(z, \partial W)} d x d y \leq C \epsilon \ell(Q)
$$

Write $f=g_{1}+g_{2}$ where $g_{1}=f \chi_{W^{*}}$. Then $g_{1}$ is the restriction to $W^{*}$ of some $f_{n}$ and so

$$
\int_{Q^{*}} \frac{g_{1}(z)}{\operatorname{dist}(z, \partial W)} d x d y \leq \int_{Q^{*}} \frac{g_{1}(z)}{1-|z|} d x d y \leq C \epsilon \ell(Q),
$$

by assumption.

To estimate the contribution of $g_{2}$, suppose $W \cap Q=Q \backslash \cup Q_{j}$. We can choose the $Q_{j}$ 's so that $\sum \ell\left(Q_{j}\right) \leq C \ell(Q)$. For each $Q_{j}, g_{2}$ is supported inside a region of width $C e^{-t} \ell\left(Q_{j}\right)$ along the base of $Q_{j}$ (because $\mu$ is supported far from $\partial W$ ), and so

$$
\int_{Q_{j}} \frac{g_{2}(z)}{\operatorname{dist}(z, \partial W)} d x d y \leq e^{-t} \ell\left(Q_{j}\right)
$$

Thus

$$
\int_{Q} \frac{g_{2}(z)}{\operatorname{dist}(z, \partial W)} d x d y \leq C\|f\|_{\infty} e^{-t} \sum_{j} \ell\left(Q_{j}\right) \leq C \epsilon \ell(Q) .
$$

As an immediate consequence we see that $f_{\mu}(W)$ is chord-arc for any $W \in \mathcal{C}$, with a constant that is independent of $W$.

Corollary 11.3. Given $\epsilon>0$ there is a $\lambda<1$ so that if $\epsilon_{k} / \epsilon_{k-1}<\lambda$ and $\left\{\mu_{n}\right\}$ satisfies the conditions of Lemma 11.2, then the following holds. Suppose $Q \in \mathcal{C}_{k, n}$. Then

$$
\sum_{Q^{\prime} \in \mathcal{C}_{k-1, n+1}} \operatorname{diam}\left(f_{\mu}\left(Q^{\prime}\right)\right) \leq \epsilon \operatorname{diam}\left(f_{\mu}(Q)\right),
$$

and

$$
\sum_{Q^{\prime} \in \mathcal{C}_{k+1, n+1}} \operatorname{diam}\left(f_{\mu}\left(Q^{\prime}\right)\right) \leq M \operatorname{diam}\left(f_{\mu}(Q)\right)
$$

Proof. The second estimate is immediate from the fact that $f_{\mu}(W(Q))$ is chord-arc. To prove the first, we note that as in the proof of Lemma 10.1, the harmonic measure of $E=\partial W \cap \cup_{\mathcal{C}_{k-1, n+1}} \partial Q$ is bounded by $A \lambda$. Since 
$f_{\mu}$ is conformal on $W$ (remember that $\mu$ is supported outside the unit disk), the same is true for the image domain. Since $\mu$ is Carleson with small norm with respect to $W, f_{\mu}(W)$ is also chord-arc and so harmonic measure is $A_{\infty}$ related to arclength. Thus $E$ has length as small as we wish if $\lambda$ is small enough. This proves the result.

\section{2. $f_{\mu}(\mathbb{T})$ has dimension 1}

We now want to show that $f_{\mu}(\mathbb{T})$ has dimension 1 . To do this we first consider the Beltrami coefficient $\sum_{k=1}^{n} \mu_{k}$ and let $f_{n}$ be the corresponding quasiconformal map fixing $0,1, \infty$.

Lemma 12.1. With $f_{n}$ as above, $f_{n}(\mathbb{T})$ is rectifiable.

Proof. To show $f_{n}(\mathbb{T})$ is rectifiable, we will estimate the length of all the image domains $f_{n}(W), W \in \mathcal{C}$ and show the total sum is finite. To each such $W$ associate a ball of twice its diameter centered at some point of $W$. Every point in $f_{n}(\mathbb{T})$ is either on the boundary of some $f_{\mu}(W)$ or is covered by infinitely many of the balls. The first set clearly has finite one dimensional measure. The second set has zero length by the following elementary observation.

Lemma 12.2. If $\sum_{n} \operatorname{diam}\left(B_{n}\right)<\infty$ and each point of $E$ is in infinitely many of the sets $\left\{B_{n}\right\}$, then $E$ has zero 1-dimensional measure.

Proof. Clearly $E$ is also covered by $\left\{B_{n}\right\}_{N}^{\infty}$ for any $N$ and for $N$ large the sum of diameters is as small as we wish.

Every domain $W$ in our decomposition of the disk has a label of the form $(k, n)$ where $k$ indicates the size of Green's function on $W$ (approximately between $\epsilon_{k-1}$ and $\epsilon_{k+1}$, except for the special cases $\left.k=1, N\right)$ and $n$ is the number of domains separating it from the origin. If $W$ has type $(k, n)$ then associated to it is a length $n$ string $s=\left\{j_{1}, \ldots, j_{n}\right\}$ where $j_{i} \in\{1, \ldots, N\}$, $j_{1}=1, j_{n}=k$ and $\left|j_{i}-j_{i+1}\right|=1$ denoting the types of domains which separate 0 from $W$. Clearly there are at most $2^{n}$ such strings of length $n$.

Lemma 12.3. Fix a string $s$ of length $n$ as above and let $\mathcal{W}(s)$ be the collection of all domains in our decomposition which have this string. Then

$$
\sum_{W \in \mathcal{W}(s)} \operatorname{diam}\left(f_{\mu}(W)\right) \leq\left(\frac{M}{\epsilon}\right)^{N / 2}(M \epsilon)^{n / 2},
$$

where $M$ and $\epsilon$ are as in Corollary 11.3 and $\epsilon \leq 1 /(4 M)$. 
Proof. A step in the string $s$ will be called "up" if it is of the form $k \rightarrow k+1$ and will be called "down" if it is of the form $k \rightarrow k-1$. Let $n_{u}$ be the number of up steps and $n_{d}$ the number of down steps in $s$. Then clearly $n_{u}+n_{d}=n$ and $n_{u} \leq n_{d}+N$. Hence $n_{d} \geq \frac{1}{2}(n-N)$. By Corollary 11.3

$$
\begin{aligned}
\sum_{W \in \mathcal{W}(s)} \operatorname{diam}\left(f_{\mu}(W)\right) & \leq M^{n_{u}} \epsilon^{n_{d}} \\
& \leq M^{N}(M \epsilon)^{n_{d}} \\
& \leq M^{N}(M \epsilon)^{\frac{1}{2}(n-N)} \\
& =\left(\frac{M}{\epsilon}\right)^{N / 2}(M \epsilon)^{n / 2} .
\end{aligned}
$$

We can now finish Lemma 12.1. The sum over all domains is bounded by

$$
\begin{aligned}
\sum_{W} \operatorname{diam}\left(f_{\mu}(W)\right) & \leq \sum_{n} \sum_{s:|s|=n} \sum_{W \in \mathcal{W}(s)} \operatorname{diam}\left(f_{\mu}(W)\right) \\
& \leq \sum_{n} \sum_{s:|s|=n}\left(\frac{M}{\epsilon}\right)^{N / 2}(M \epsilon)^{n / 2} \\
& \leq\left(\frac{M}{\epsilon}\right)^{N / 2} \sum_{n} 2^{n}(M \epsilon)^{n / 2} \\
& \leq 2(M / \epsilon)^{N / 2}
\end{aligned}
$$

if $M \epsilon<1 / 4$. This completes the proof.

Now we can prove that $f_{\mu}(\mathbb{T})$ has dimension 1 if $\mu$ is chosen appropriately.

Lemma 12.4. Suppose notation is as above. Suppose $\varphi(t):[0, \infty) \rightarrow \infty$ is increasing, continuous and satisfies $\varphi(t)=o(t)$. There is a sequence $r_{n} \nearrow \infty$ so that if $\rho\left(\operatorname{supp}\left(\mu_{n}\right), \operatorname{supp}\left(\mu_{n+1}\right)\right) \geq r_{n}$, then $\mathcal{H}^{\varphi}\left(f_{\mu}(\mathbb{T})\right)=0$.

Proof. Suppose we have already chosen $\mu_{1}, \ldots, \mu_{n}$. Then by Lemma 12.1, $f_{n}(\mathbb{T})$ is rectifiable. Thus there is a cover of $f_{n}(\mathbb{T})$ by open balls $\left\{B\left(x_{j}, r_{j}\right)\right\}$ such that $\sum_{j} \varphi\left(r_{j}\right) \leq 2^{-n}$. Then $U_{n}=\cup_{j} B\left(x_{j}, r_{j}\right)$ is an open neighborhood of $f_{n}(\mathbb{T})$ and if $r_{n}$ is large enough we have $f_{\mu}(\mathbb{T}) \subset U_{n}$ as well (since $f \rightarrow f_{n}$ uniformly on compact sets as $\left.r_{n} \rightarrow \infty\right)$. This proves the lemma.

Taking $\varphi(t)=t \log (1 / t)$, for example, and $\mu$ satisfying the lemma shows that $f_{\mu}(\mathbb{T})$ can have dimension 1 , as desired. 


\title{
References
}

[1] Astala, K. And Zinsmeister, M.: Mostow rigidity and Fuchsian groups C. R. Acad. Sci. Paris Sér. I Math. 311 (1990), 301-306.

[2] Bishop, C. J.: Quasiconformal mappings of $Y$-pieces. Rev. Mat. Iberoamericana 18 (2002), 627-652.

[3] Bishop, C. J.: Divergence groups have the Bowen property. Ann. of Math. 154 (2001), 205-217.

[4] Bishop, C. J. And Jones, P.W.: Compact deformations of Fuchsian groups. To appear in J. Anal. Math.

[5] Bishop, C. J. And Jones, P.W.: Harmonic measure, $L^{2}$ estimates and the Schwarzian derivative. J. Anal. Math. 62 (1994), 77-113.

[6] Bowen, R.: Hausdorff dimension of quasicircles. Inst. Hautes Études Sci. Publ. Math. 50 (1979), 11-25.

[7] Garnett, J.B.: Bounded analytic functions. Academic Press, 1981.

[8] Jerison, D.S. And Kenig, C.E.: Hardy spaces, $A_{\infty}$ and singular integrals on chord-arc domains. Math. Scand. 50 (1982), 221-248.

[9] Jones, P.W.: Rectifiable sets and travelling salesman problem. Invent. Math. 102 (1990), 1-15.

[10] Keen, L.: Collars on Riemann surfaces. In Discontinuous groups and Riemann surfaces, Ann. of Math. Stud. 79, Princeton Univ. Press, Princeton, N.J., 1974, 263-268.

[11] Matelski, J. P.: A compactness theorem for Fuchsian groups of the second kind. Duke Math. J. 43 (1976), no. 4, 829-840.

[12] Pommerenke, Ch.: Boundary behaviour of conformal maps. Grundlehren Math. Wiss. 299, Springer-Verlag, Berlin, 1992.

[13] Semmes, S.: Quasiconformal mappings and chord-arc curves. Trans. Amer. Math. Soc. 306 (1988), 233-263.

Recibido: 29 de noviembre de 2000

\author{
Christopher J. Bishop ${ }^{1}$ \\ Mathematics Department \\ SUNY at Stony Brook \\ Stony Brook, NY 11794-3651 \\ bishop@math.sunysb.edu
}

\footnotetext{
${ }^{1}$ Partially supported by NSF Grant DMS 0103626
} 